\title{
What kind of priming is most effective in the processing of relative clauses in context?
}

\author{
Zhaohong Wu \& Alan Juffs*
}

\begin{abstract}
This paper examines the influence of different kinds of preceding contexts on the processing of Chinese relative clauses (RCs). We systematically compared the processing of RCs in canonical, non-canonical, and "null" contexts. This paper is the first to systematically examine three accounts of priming (the thematic pattern priming account proposed by Lin (2014), in addition to both the verb phrase constituent priming account and the syntactic position sequence priming account proposed by Fedorenko, Piantadosi, and Gibson (2012)) in RC processing. Results showed discrepancies between predictions from each priming account and the actual results. None of the three priming accounts could sufficiently explain the results in Chinese. Alternative possible explanations were suggested, including: (1) having a context makes RC reading more natural and frequency effects less obvious; (2) the NPs inside the RCs are primed by the original thematic roles or grammatical functions of same NPs in the critical context sentence; (3) an interplay of all three different kinds of priming in the processing of RCs in context may occur.
\end{abstract}

Keywords. priming; processing; relative clauses; Chinese; preceding context; thematic pattern

1. Introduction. One of the goals in the sentence processing literature is to uncover the constraints on online parsing of a sentence (e.g., the constraint satisfaction mechanisms in MacDonald, Pearlmutter \& Seidenberg (1994)) in order to reveal the principles underlying realtime language processing and cognition. RCs have received a great deal of interest in the psycholinguistic literature in exploring constraints on online sentence processing because they exhibit complex structural relationships that can provide a window into processing. Research in English $\mathrm{RC}$ processing has shown a consistent subject relative clause (SRC) advantage (e.g., Fedorenko, Piantadosi \& Gibson 2012, Gibson, Desmet, Grodner, Watson \& Ko 2005). This has led to a series of explanations, including experience- and surprisal-based theories (Gennari \& MacDonald 2008, Levy 2008), memory-based accounts which include storage cost accounts and integration/retrieval cost accounts (Gibson 1998, 2000, Lewis \& Vasishth 2005, Warren \& Gibson 2002), differences in canonical vs. non-canonical word order accounts (MacDonald \& Christiansen 2002, Tabor, Juliano \& Tanenhaus 1997), the noun phrase accessibility hierarchy (NAPH) account (Keenan \& Comrie 1977), perspective shift (MacWhinney 1977, 1982, MacWhinney \& Pléh 1988), and the entropy reduction account (Yun, Chen, Hunter, Whitman \& Hale 2015).

However, the existing literature on the processing of Chinese RCs has led to mixed results. The majority of previous studies have reported that SRCs are easier to process than object relative clauses (ORCs) (Jäger, Chen, Li, Lin \& Vasishth 2015, Lin \& Bever, 2006, Vasishth, Chen, Li \& Guo 2013, Wu, Kaiser \& Anderson 2012), while others have shown that ORCs are easier to process than SRCs (Hsiao \& Gibson 2003, Gibson \& Wu 2013, Packard, Ye \& Zhou 2011, Vasishth, Chen, Li \& Guo 2013). Chinese provides an interesting case to test different accounts in the processing of RCs because Chinese, unlike most other languages, has an SVO word order but

\footnotetext{
* Authors: Zhaohong Wu, University of Pittsburgh (zhw39@pitt.edu) \& Alan Juffs, University of Pittsburgh (juffs@pitt.edu).
} 
also a head-final RC structure. Consider the examples of Chinese SRC and ORC in (1) (taken from Hsiao \& Gibson 2003 and reused in Gibson \& Wu 2013).

a. SRC

- 邀请 富豪 的 官员 心怀 不轨。
- yaoqing fuhao de guanyuan xinhuai bugui
invite tycoon REL official heart have bad
'The official who invited the tycoon had bad intentions.'

b. ORC

富豪 邀请 - 的 官员 心怀 不轨。
fuhao yaoqing - de guanyuan xinhuai bugui
tycoon invite - REL official heart have bad
'The official who the tycoon invited had bad intentions.'

(1a) and (1b) are two sentences with restrictive RCs that modify the subject of the entire sentence, guanyuan ('the official'). The underline marks the RC gap position. The sentence in (1a) contains a SRC in which yaoqing ('invite') is the verb, and fuhao ('tycoon') is the object of the verb yaoqing ('invite'), de is the RC marker (the relativizer, indicating the existence of a $\mathrm{RC}$ ), and the head noun guanyuan ('the official'), which functions as the subject of the verb in the RC. The sentence in (1b) contains an ORC, in which yaoqing ('invite') is again the verb, and fuhao ('tycoon') is the subject of the verb yaoqing ('invite'), de is again the RC marker, and the head noun guanyuan ('the official') functions as the object of the verb in the RC. The SRC in (1a) and the ORC in (1b) are both subject-modifying RCs in that they modify the subject of the main clause, guanyuan ('the official'). Object-modifying RCs, RCs that modify the object of the main clause, are beyond the scope of this study.

Most previous experiments on Chinese $\mathrm{RC}$ processing have been run without a preceding context (Hsiao \& Gibson 2003, Jäger, Chen, Li, Lin \& Vasishth 2015, Lin \& Bever 2006, Packard, Ye \& Zhou 2011, Wu, Kaiser \& Anderson 2012). However, the essential role of a RC is to modify and thus identify one out of many possible referents. A great deal of evidence has shown the strong influence of context in sentence processing and interpretation (see Fedorenko, Piantadosi \& Gibson 2012 for a brief summary). Therefore, exploration of RC processing justifies the natural presentation of RCs in an appropriate context.

The first study of the processing of Chinese RCs in a context was conducted by Gibson and $\mathrm{Wu}$ (2013). If a reader reads from the very beginning of the ORC construction until the position of the verb in the RC, he/she is very likely to be expecting the object of the verb so as to complete a simple SVO sentence. However, on encountering the relativizer $d e$, the string must be reinterpreted as an ORC within a main clause. They manipulated the context so that participants were primed by the supportive context to have an expectation for an incoming RC construction. The temporary ambiguity in the initial reading of an ORC could therefore be reduced, if not eliminated completely. Their finding of an ORC advantage at the head noun was explained from the perspective of an integration account, which has also successfully predicted a SRC advantage in English.

Hsiao and Gibson's (2003) study, which originally found an overall ORC advantage without a context, was run again by Vasishth, Chen, Li, and Guo (2013). Using maximal linear mixed effects models, with negative reciprocal transformation of raw reading times (determined by the Box-Cox procedure) in order to satisfy the normality assumption of residuals. Vasishth et al. (2013) found instead a SRC advantage at the headnoun, not an ORC advantage. They also repli- 
cated Gibson and Wu's study and did find a marginally significant ORC advantage at both the relativizer and the head noun. However, Vasishth argued that in Gibson and Wu (2013) "there are a few items in subject relative only that trigger high reading times, which lead to unequal variances in subject and object relatives at the head noun that are driving the results in the published study (personal communication to Zhaohong Wu via email, Nov 25th 2013)". Vasishth et al. (2013) argued that this finding of an ORC advantage at the head noun is consistent with the working memory storage/integration accounts which have been successful in accounting for the SRC advantage in English, but inconsistent with the frequency-based accounts which have also been successful in accounting for the SRC advantage in English, since SRCs have been found to more frequent than ORCs in both Chinese and English in various corpus studies (e.g., Reali \& Christiansen 2007 for English; Vasishth et al. 2013 for Chinese). They suggested the possibility that the ORC advantage at the head noun was a spillover effect from the preceding region. Assuming that the supportive context would eliminate the temporary ambiguity, they argued that the ORC advantage at the relativizer position could not be accounted for by either the frequencybased accounts or the storage/integration accounts.

Packard, Ye, and Zhou (2011) carried out an ERP study on Chinese RC processing without a context and found an ORC advantage for subject-modifying RCs at the relativizer de demonstrated by the ERP P600 measure, which has been claimed to reflect syntactic processing. They attribute this result to a greater syntactic (filler-gap) integration cost for the SRC condition than the ORC condition at the relativizer, but they did not find an SRC vs. ORC difference at the head noun demonstrated by the P600 measure. Their assumption that the filler-gap integration happens at the relativizer position for subject-modifying RCs seems to be able to account for the ORC advantage in the P600 measure in their study and the ORC advantage at the relativizer position found in Vasishth et al. (2013). This study suggests that the supportive context in Gibson and Wu's (2013) study and in Vasishth et al.'s (2013) replication of their study may not have helped significantly in priming participants to confidently predict an incoming RC, because integration seemed to happen at the relativizer position even without a preceding context in Packard et al. (2011).

Finally, Vasishth et al. (2013) also performed a Bayesian meta-analysis of previous studies on Chinese relative clauses and found that the posterior probability of finding a SRC advantage of approximately $78-80 \%$. They therefore argued against the working memory storage/integration account of RC processing, in favor of the frequency account.

To reconcile all these contradictory findings, Vasishth et al. (2013) suggested Lin's (2010, 2014) account might be plausible. Lin $(2010,2014)$ argued that thematic pattern priming might lead to the finding of an ORC advantage in Gibson and Wu (2013). SRCs in Chinese have the thematic pattern of VERB-PATIENT-AGENT (in which case, the AGENT is the head noun), while ORCs have the thematic pattern of AGENT-VERB-PATIENT (in which case, the PATIENT is the head noun). For example, the thematic pattern in (1a) for a Chinese SRC is VERB ('invite') - PATIENT ('tycoon') -AGENT ('official'), whereas the thematic pattern in (1b) for a Chinese ORC is AGENT ('tycoon') - VERB ('invite') - PATIENT ('official').

Lin $(2010,2014)$ manipulated the thematic patterning of the critical context sentence in the preceding context in two ways, an example of each is given in (2) and (3). First, consider the example of context manipulation in (2) (adapted from Gibson \& Wu, 2013), where the thematic patterning in the critical context sentence in the preceding context has the consistent canonical AGENT-VERB-PATIENT thematic pattern (A verbed B, and then B verbed another A). Lin found no significant difference between SRCs and ORCs on the critical regions (the relativizer 
de and the head noun) when the critical context sentence in the preceding context has the consistent AGENT-VERB-PATIENT thematic pattern. Next, consider (3) (modified from (2), in which only the critical context sentence is shown here because it is the only difference between the two contexts). Note that the critical context sentence in the preceding context has the consistent non-canonical AGENT-PATIENT-VERB thematic pattern. This construction is the $b a-$ construction which places the theme NP before the verb: A $b a$ B verbed, and then B $b a$ another A verbed. Lin $(2010,2014)$ did not find a significant difference between SRCs and ORCs on the critical regions (the relativizer $d e$ and the head noun) when the preceding context has the consistent AGENT-PATIENT-VERB thematic pattern. However, in the spillover (the first region after the head noun), Lin found a significant main effect of RC type (ORCs faster than SRCs), but no significant main effect of context or their interaction. On the second region after the head noun, Lin found no significant main effects but a significant interaction, such that ORCs were significantly faster only in the canonical context condition, while there was no reliable difference between ORCs and SRCs in the non-canonical context condition.

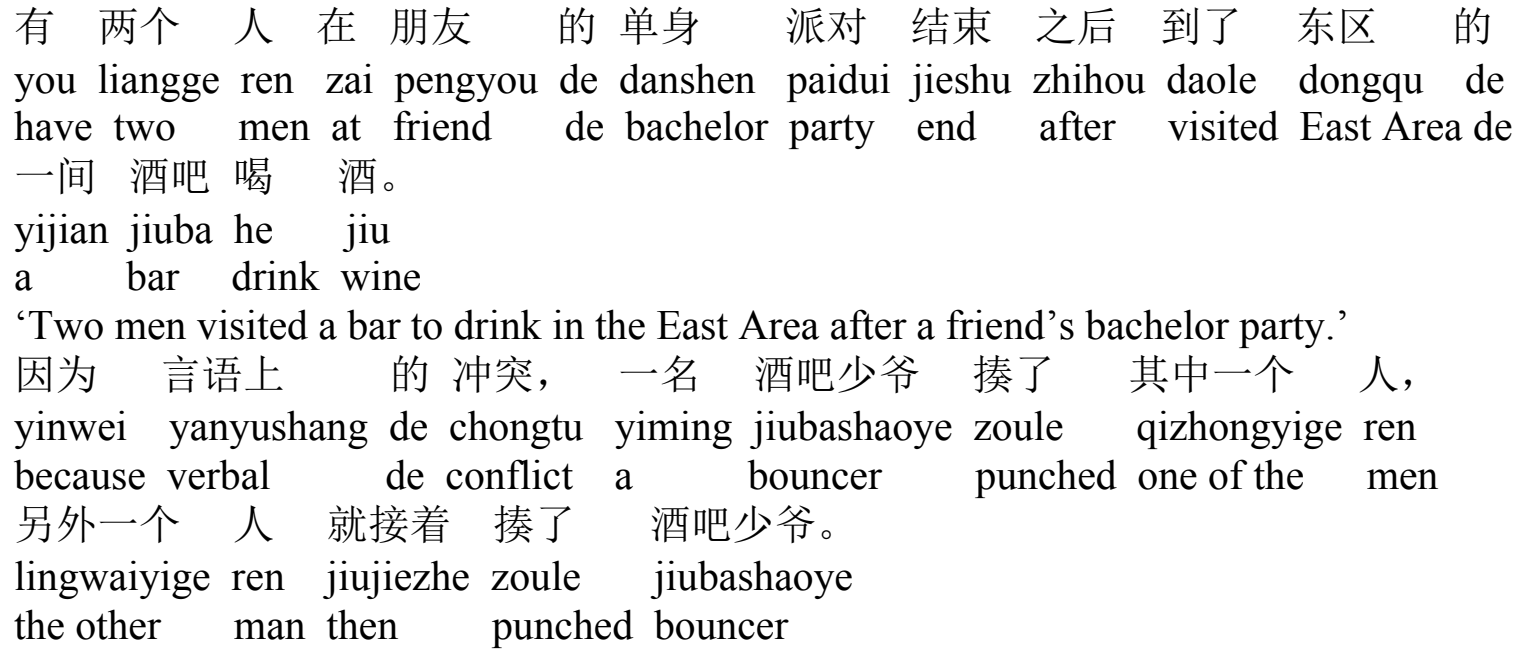
other. Which man had the bar owner met?'

$\mathrm{ORC}$ : 小美说: 酒吧少爷 揍的人是老板见过的。 Xiaomei shuo: jiubashaoye zou de ren shi laoban jianguo de Xiaomei say: bouncer punch REL man be owner met de 'Xiaomei said: the man who the bouncer punched is who the owner met.'

$\mathrm{SRC}$ : 小美 说: 揍酒吧少爷的人是老板见过的。 Xiaomei shuo: zou jiubashaoye de ren shi laoban jianguo de Xiaomei say: punch bouncer REL man be owner met de 'Xiaomei said: the man who punched the bouncer is who the owner met.' 


$$
\begin{aligned}
& \text { 因为言语上 的冲突, 一名 酒吧少爷 把其中一个 人 揍了, } \\
& \text { yinwei yanyushang de chongtu yiming jiubashaoye ba qizhongyige ren zoule } \\
& \text { because verbal de conflict a bouncer ba one of the men punched } \\
& \text { 另外一个 人 就接着 把酒吧少爷 揍了。 } \\
& \text { lingwaiyige ren jiujiezhe ba jiubashaoye zoule } \\
& \text { the other man then ba bouncer punched } \\
& \text { 'Because of some verbal conflicts, a bouncer punched one of the men, and then another } \\
& \text { man punched the bouncer.' }
\end{aligned}
$$

Lin also tested the SRC/ORC advantage when the critical context sentence in the preceding context follows the structure "A verbed B, and then another A bei B verbed" and thus has a thematic pattern ordering of AGENT-VERB-PATIENT and then PATIENT-AGENT-VERB (which is the passive bei-construction in Chinese). He did not find any difference on the critical regions or any regions following the head noun. Consider (4) (modified from (2), only the second sentence is shown here because it is the only difference between the two contexts).

因为言语上 的冲突，其中一个 人 揍了 一名 酒吧少爷,
yinwei yanyushang de chongtu qizhongyige ren zoule yiming jiubashaoye
because verbal de conflict one of the men punched a bouncer
另外一个 人 就接着 被 酒吧少爷 揍了。
lingwaiyige ren jiujiezhe bei jiubashaoye zoule
the other man then bei bouncer punched
'Because of some verbal conflicts, one of the men punched a bouncer, and then another
man was punched by the bouncer.'

To summarize, Lin's thematic priming hypothesis was able to account for interaction between RC type and context in the second region after the head noun, such that there was an ORC advantage in the canonical context condition but no $\mathrm{RC}$ complexity effect in the non-canonical context condition. However, Lin's thematic priming account cannot explain his finding of an ORC advantage in the non-canonical $b a$-construction context condition on the first region after the head noun, because the critical context sentence in the non-canonical ba-construction context condition has a thematic pattern ordering AGENT-PATIENT-VERB that is different from both the thematic pattern orderings of SRCs and ORCs in Chinese.

Fedorenko, Piantadosi, and Gibson (2012) also compared processing of English RCs in a supportive canonical context. An example is provided in (5), directly from Fedorenko et al. (2012). They also included a null context, which was created by omitting the supportive context, starting with Mary's utterance. The supportive context condition consists of two sentences that set up a scenario: Mary's utterance which contains a RC from which reading times are drawn, and John's response, which either agrees with Mary ("Yeah, that's right") or disagrees with Mary ("I am not sure about that). On the RC region, Fedorenko et al. (2012) found a significant SRC advantage, and a significant main effect of context such that RCs in the supportive context condition were processed faster than those in the null context condition, but no interaction was found between $\mathrm{RC}$ type and context, although the RC complexity effect was numerically larger in the supportive context. On the following main verb region, there were significant effects of $\mathrm{RC}$ type (SRC advantage), context, and also an interaction between the two, such that there was no RC effect for the null context condition but there was a reliable RC effect (SRC advantage) 
for the supportive context condition. Fedorenko et al. (2012) suggested two lexico-syntactic priming accounts for the larger $\mathrm{RC}$ complexity effect in the supportive context condition than in the null context condition. First, the verb phrase constituent priming account suggests that if there is the same corresponding verb phrase constituent in the context, it can prime the sequence corresponding to that in the relative clause, e.g., the verb phrase "attacked the senator" in the context will prime the corresponding sequence in the SRC ("who attacked the senator") that preserves the verb phrase constituent but not that in the ORC ("who the senator attacked") that does not preserve the verb phrase constituent. Second, the syntactic position sequence priming account suggests that the sequences of syntactic positions are primed together with their associated lexical items, such that if the context contains subject-verb-object sequences, which are the same as in SRCs, but not object-subject-verb sequences as in ORCs, SRCs but not ORCs are primed.

At the press-conference, a senator and two reporters got into an argument.

The senator [attacked] $]_{\text {VERB }}$ [one of the reporters $]_{\text {PATIENT }}$ and then [the other reporter $]_{\text {AGENT }}$ [attacked] VERB $[\text { the senator }]_{\text {PATIENT. }}$.

SRC: Mary: I heard that [the reporter $]_{\text {AGENT }}$ that [attacked] $]_{\text {VERB }}[\text { the senator }]_{\text {PATIENT }}$ admitted to making an error.

ORC: Mary: I heard that [the reporter $]_{\text {PATIENT }}$ that [the senator $]_{\text {AGENT }}$ [attacked $]_{\text {VERB }}$ admitted to making an error.

John: I'm not sure about that.

Lin's (2010) thematic pattern priming account may also explain the larger RC extraction effect in the supportive context in Fedorenko et al. (2012), as the preceding context used in Fedorenko et al. (2012) has the same thematic pattern AGENT-VERB-PATIENT as that in English SRCs. If thematic pattern priming is correct, then English $\mathrm{RC}$ processing in a context with the consistent thematic pattern ordering of PATIENT-VERB-AGENT ( $\mathrm{A}$ is verbed by $\mathrm{B}$, and then $\mathrm{B}$ is verbed by another A, as in Example (6)) might induce an SRC advantage that is smaller (or at least to the same extent) than a null context would. This is because the critical context pattern is inconsistent with the SRC AGENT-VERB-PATIENT pattern. It should definitely induce a smaller SRC advantage than the supportive canonical context used in Fedorenko et al. (2012).

At the press-conference, a senator and two reporters got into an argument.

[One of the reporters $]_{\text {PATIENT }}$ was [attacked] $]_{\text {VERB }}$ by [the senator $]_{\text {AGENT }}$ and then

[the senator $]_{\text {PATIENT }}$ was [attacked] $]_{\text {VERB }}$ by [the other reporter $]_{\text {AGENT }}$.

SRC: Mary: I heard that [the reporter] $]_{\text {AGENT }}$ that [attacked] $]_{\text {VERB }}$ [the senator $]_{\text {PATIENT }}$ admitted to making an error.

ORC: Mary: I heard that [the reporter $]_{\text {PATIENT }}$ that [the senator $]_{\text {AGENT }}$ [attacked $]_{\text {VERB }}$ admitted to making an error.

John: I'm not sure about that.

Therefore, Lin's thematic pattern priming account makes the same prediction as Fedorenko et al.'s (2012) syntactic position sequence account in the canonical AGENT-VERB-PATIENT context, but they differ in their predictions in the non-canonical PATIENT-VERB-AGENT (A is verbed by B, and then B is verbed by another A) in English. This is because Lin's $(2010,2014)$ thematic pattern priming would predict a smaller SRC advantage than the canonical AGENTVERB-PATIENT context, but Fedorenko et al.'s (2012) syntactic position sequence priming account would not predict a difference between the two types of contexts, since in both contexts the syntactic position sequence is subject-verb-object. In other words, the crucial difference in 
claims pits a semantic ordering of arguments (AGENT, PATIENT, etc.) versus a purely syntactic ordering (subject, object, etc.). Note that a grammatical function of Subject can be either a PATIENT (passive) or an Agent (active). Therefore, it is possible to test which account can better explain English relative clause processing in the non-canonical PATIENT-VERB-AGENT context ( $\mathrm{A}$ is verbed by B, and then B is verbed by another A) in English. Fedorenko et al.'s (2012) verb phrase constituent priming account, however, would make the same prediction of an SRC advantage in Chinese and English since the SRCs keep the verb phrase constituent intact, contrary to ORCs. This runs counter to Gibson and Wu's finding of an ORC advantage in the canonical AGENT-VERB-PATIENT context.

Manipulating the preceding context thematic pattern permits the testing these three types of priming hypotheses: the thematic pattern priming account, the verb phrase constituent priming account, and the syntactic position sequence account. Quite apart from these theoretical considerations, the controversy in the previous literature regarding the SRC vs. ORC asymmetry in Chinese calls for a more systematic study with better manipulation of the materials. Only Gibson and $\mathrm{Wu}$ (2013) and Vasishth et al. (2013) focused on RCs in a canonical context, while Lin (2010, 2014) compared RCs in canonical and non-canonical contexts. The current study is the first to systematically compare the processing of RCs in a canonical, a non-canonical context, and a "null" context in Chinese and English in one experiment and the first to systematically examine the three accounts of priming in RC processing in both English and Chinese. However, due to space limitations, this paper reports the findings of Chinese $\mathrm{RC}$ processing only.

2. Experiment. The current experiment was designed to further replicate the SRC vs. ORC asymmetry in Chinese, while manipulating the different types of context, and to examine the three priming hypotheses. For Chinese, the different contexts included a "null" context, an item example (translated into English) of which is given in (7), a canonical AGENT-VERBPATIENT context, as in (2), and a non-canonical AGENT-PATIENT-VERB context (A ba B verbed, and then $B$ ba another A verbed), as in Example (3). The different accounts of priming lead to different predictions in a particular type of context.

(7) The TA and several students are having a review session.

The TA gave the students some exercises for them to practice.

John said: I hear that they have some disagreement over the right answer to one of the questions.

They all have completely different answers from each other.

ORC: Mary said: The student that the TA opposes complains about the TA's incompetence.

SRC: Mary said: The student that opposes the TA complains about the TA's incompetence.

\footnotetext{
${ }^{1}$ Here, "null" is in quotation marks to indicate that the context is not exactly null, but that the participants would not be primed by the context such that they would expect reading an upcoming RC as they would be by the canonical or non-canonical context. The critical context sentence in the "null" context condition is just a general situational sentence that does not introduce hypothesized thematic pattern priming or verb phrase priming or syntactic positions sequence priming as the critical context sentences in the canonical context or non-canonical context conditions do. Therefore, the "null" context used here in this study is different from the null context used in previous literature only in the fact that there is a context that does not induce priming effects. The "null" context is chosen over the null context condition in this study in order to remain the consistency and similarity of the items.
} 
2.1. DeSIGN AND MATERIALS. The experiment contained canonical, non-canonical, and "null" contexts (48 items total) and 48 fillers. Each context condition has 16 sets of items (each set of items has two conditions: either an SRC or an ORC in the critical sentence). The critical items in the experiment all follow the same format: the first sentence is a general situation sentence; the second is a critical context sentence that hypothetically induced either (i) thematic pattern priming or (ii) verb phrase priming or (iii) syntactic position sequence priming. The structure of the critical context sentence is counterbalanced with regard to whether the description started with the unique noun or the non-unique noun, following Fedorenko et al. (2012); the third is a statement by "Xiaoming" which starts with "I hear/heard that"; the fourth is either a question or a statement by "Xiaoming"; and the last sentence is the target sentence, a statement by "Xiaomei" that contains the RC lies and in which critical reading times are measured.

The 48 fillers followed the same format as the critical target materials: two context sentences, a statement by "Xiaoming", followed by a response from "Xiaomei". Xiaomei's response in the filler materials contained no RCs. An example is given in (8) (from Gibson \& Wu 2013).

$$
\begin{aligned}
& \text { zai yige dade gongyu zhuzhaiqu li, you yige xiaofangyuan cong dahuo zhong jiule } \\
& \text { yiming furen. } \\
& \text { 'A firefighter saved a woman from a fire in a large apartment complex.' } \\
& \text { ta shi ge yisheng, erqie houlai faxian ta juran shi tade gaozhong tongxue. } \\
& \text { 'She was a doctor, and she turns out to be his high-school classmate.' } \\
& \text { Xiaoming shuo: wo tingshuo tamen shangge yue jiehunle. } \\
& \text { 'Xiaoming said: I heard that they got married last month.' } \\
& \text { Xiaomei shuo: na zhen shi langman a, ta jia gei le tade yingxiong le. } \\
& \text { 'Xiaomei said: That's very romantic. She got married to her hero.' }
\end{aligned}
$$

Some of the materials were adapted from Hsiao \& Gibson (2003), Gibson \& Wu (2013), Fedorenko et al. (2012), and Xu (2014), and the others were designed for the experiment. The materials were adapted and designed in consideration of naturalness, consistency in the grammatical function of the head noun (Gibson and Wu's did not strictly control for the grammatical function of the head noun), and animacy (Traxler, Morris \& Seely 2002, Traxler, Williams, Blozis \& Morris 2005, Wu, Kaiser \& Anderson 2012). All the critical nouns were animate and all RCs were subject-modifying.

SRCs in Chinese follow the thematic pattern of VERB-PATIENT-AGENT and the syntactic position sequence of verb-object-subject; SRCs contain an intact VP. ORCs in Chinese follow the thematic pattern of AGENT-VERB-PATIENT and the syntactic position sequence of subject-verb-object; in the ORC the VP is not intact, but is rather separated by the relativizer $d e$.

With regard to the predictions from the different priming accounts, for the "null" context, since the "null" context does not have a critical context sentence that introduces priming, all accounts would predict an SRC advantage, according to the previous literature on Chinese RC processing.

For the supportive canonical context (AGENT-VERB-PATIENT; subject-verb-object; VP intact), the thematic pattern priming account would predict an ORC advantage, because the thematic pattern in the critical sentence context (AGENT-VERB-PATIENT) is the same as that in Chinese ORCs (AGENT-VERB-PATIENT), but not in Chinese SRCs (VERB-PATIENTAGENT); the VP constituent priming account would predict a larger SRC advantage than the "null" context, since VP is intact in the critical context sentence and also in the Chinese SRCs, but not in Chinese ORCs; and the syntactic position sequence priming account would predict an 
ORC advantage, since the syntactic position sequence in the critical sentence context (subjectverb-object) is the same as that in Chinese ORCs (subject-verb-object), but not that in Chinese SRCs (verb-object-subject).

For the non-canonical $b a$-construction context (AGENT-PATIENT-VERB; subject-objectverb; VP separated), the thematic pattern priming account would predict no difference between SRCs and ORCs, following Lin's $(2010,2014)$ results, or a smaller SRC advantage than the "null" context, since neither Chinese SRCs (VERB-PATIENT-AGENT) nor ORCs (AGENTVERB-PATIENT) follow the thematic pattern in the non-canonical context (AGENT-PATIENTVERB); the VP constituent priming account might predict no difference between SRCs and ORCs, or a smaller SRC advantage than the "null" context, since the critical context sentence does not have an intact VP such that no VP constituent priming will be possible, and the syntactic position sequence priming account might predict no difference between SRCs and ORCs, or a smaller SRC advantage than the "null" context, since neither Chinese SRCs (verb-object-subject) nor ORCs (subject-verb-object) follow the syntactic position sequence in the non-canonical context (subject-object-verb).

2.2. PARTICIPANTS. 46 native Mandarin Chinese native speakers (20 undergraduate students and 26 graduate students at University of Pittsburgh or Carnegie Mellon University) were recruited for the Chinese version of the experiment. All participants were paid for their participation. All participants were naive to the theoretical purposes of the study.

2.3. Procedure. The task was self-paced reading, using a moving window display (Just, Carpenter \& Woolley 1982), run on Linger 2.88 developed by Doug Rohde. The context was a sentence-by-sentence presentation and the target sentence was a word-by-word presentation. A comprehension question regarding the item/filler content was asked at the end of each trial in order to make sure that participants read the sentences carefully enough for meaning. For both the items and the fillers, half of the comprehension questions asked about the content of the context sentences and the other half asked about the final clause (the response from Xiaomei). Participants pressed F or J to respond "yes" or "no". Feedback was presented briefly on the screen if they gave the wrong answer to the comprehension question.

2.4. READING TIME RESULTS. The critical regions in the critical target sentences for the Chinese experiment were $\mathrm{VN} / \mathrm{NV}$, de, headnoun (head noun), mainverb (main verb), mainverb+1 (the region after the main verb), and I also combined de and headnoun together to form a $d e+$ headnoun region, rendering six critical regions total.

Figure 1 shows the line graph of mean raw reading times per region per condition. Linear mixed effects models with varying intercepts and slopes (including the varying intercept and slope correlation) for the random effects of items and subjects were fit in R (R Core Team 2014) using the lme4 package (Bates, Maechler, Bolker \& Walker 2014) on negative reciprocal transformed reading times on each critical region, with Context (dummy coded, with the "null" context as the reference group), RC Type (whether it is an SRC, coded as -0.5 , or an ORC, coded as 0.5 ) and their interaction as fixed effects. The variable Context was recoded into "canonical" (comparing canonical and "null" contexts) and "non-canonical" (comparing non-canonical and "null" contexts). 


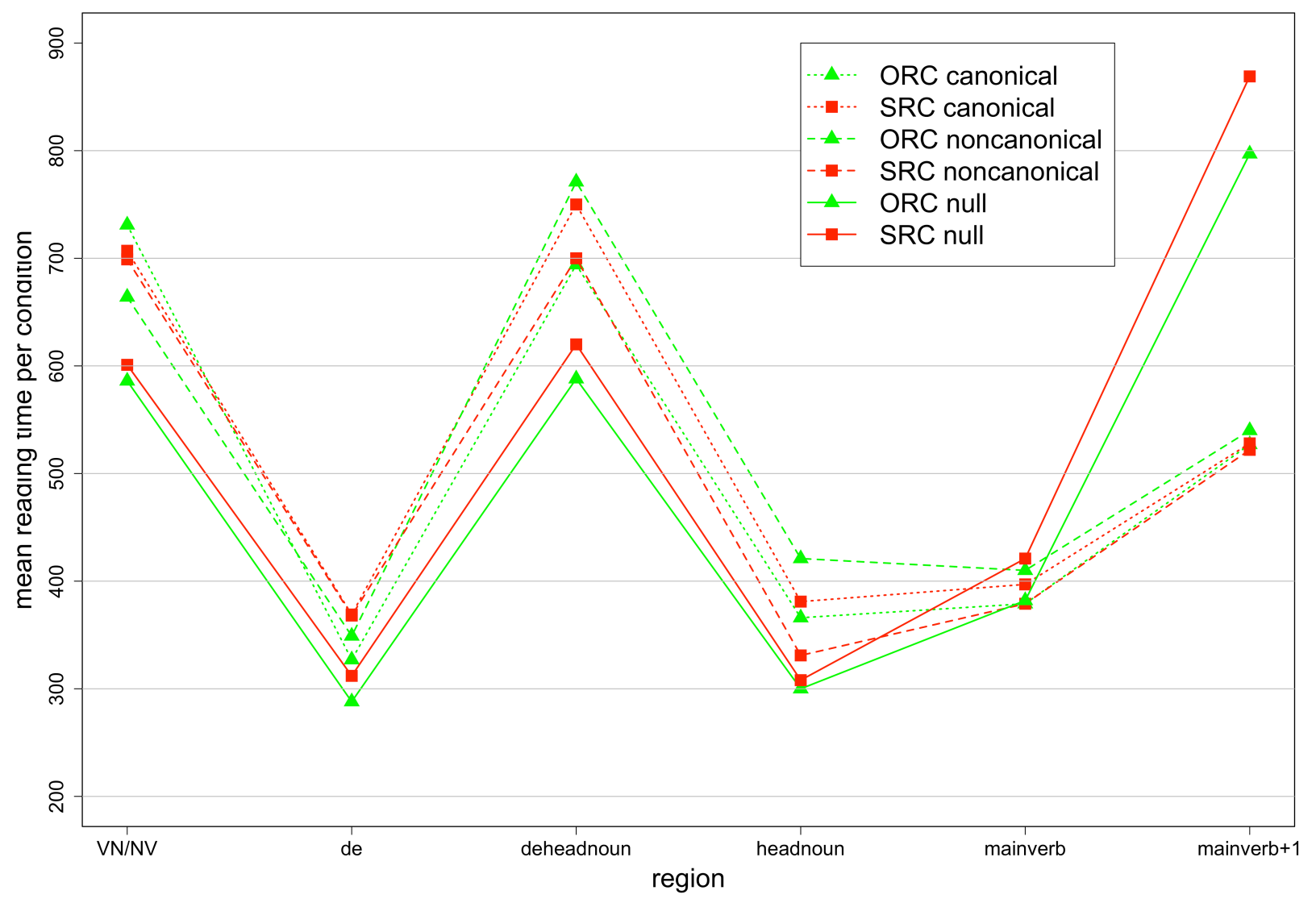

Figure 1. Mean raw reading times per region in different conditions for the Chinese dataset

Analysis on the VN/NV region revealed (a) no significant main effect of RC Type ( $t=-1.66$, $p>.5$ ), such that SRCs were slower than ORCs but not significant in the "null" context; (b) a significant main effect of "canonical" $(t=5.07, p<.001)$, such that the canonical context conditions were processed slower than the "null" context conditions; (c) a significant main effect of "non-canonical" $(t=4.38, p<.001)$, such that the non-canonical context conditions were processed slower than the "null" context conditions; (d) a significant interaction between "canonical" and RC Type $(t=2.86, p<.01)$, such that SRCs were faster than ORCs in the canonical context; (e) but no interaction between "non-canonical" and RC Type $(t=0.24, p>.5)$.

Analysis on the de region revealed (a) a significant main effect of RC Type $(t=-1.750, p<$ .05 ), such that SRCs were significantly slower than ORCs in the "null" context; (b) a significant main effect of "canonical" $(t=3.046, p<.001)$, such that the canonical context conditions were processed slower than the "null" context conditions; (c) a significant main effect of "noncanonical" $(t=3.057, p<.001)$, such that the non-canonical context conditions were processed slower than the "null" context conditions; (d) but no significant interaction between "canonical" and $\mathrm{RC}$ Type $(t=0.332, \mathrm{p}>.5)$ or between "non-canonical" and $\mathrm{RC}$ Type $(\mathrm{t}=0.195, \mathrm{p}>.5)$ such that there was an ORC advantage in both canonical and non-canonical context conditions.

Analysis on the headnoun region revealed (a) no significant main effect of RC Type ( $t=$ $1.680, p>.1$ ), such that SRCs were slower than ORCs but not significant in the "null" context; (b) a significant main effect of "canonical" $(t=2.128, p<.05)$, such that the canonical context 
conditions were processed slower than the "null" context conditions; (c) no significant main effect of "non-canonical" ( $t=1.855, p>.1)$; (d) and no significant interaction between "canonical" and RC Type $(t=0.258, p>.5)$ and no interaction between "non-canonical" and RC Type $(t=$ $1.941, p>.05)$ such that there was no significant effect of RC Type in either canonical or noncanonical contexts.

Analysis on the $d e+$ headnoun region revealed (a) a significant main effect of RC Type ( $t=$ 2.146, $p<.05$ ), such that SRCs were significantly slower than ORCs in the "null" context; (b) a significant main effect of "canonical" $(t=2.857, p<.01)$, such that the canonical context conditions were processed slower than the "null" context conditions; (c) a significant main effect of "non-canonical" $(t=2.793, p<.01)$, such that the non-canonical context conditions were processed slower than the "null" context conditions; (d) but no significant interaction between "canonical" and RC Type $(t=0.327, p>.5)$ or between "non-canonical" and RC Type $(t=$ $1.200, p>.1)$ such that there was a significant ORC advantage in both canonical and noncanonical contexts.

Analysis on the mainverb region revealed (a) a significant main effect of RC Type ( $t=-$ $2.370, p<.05$ ), such that SRCs were processed slower than ORCs in the "null" context; (b) no significant main effect of "canonical" $(t=-0.711, p>.1)$ (c) a significant main effect of "noncanonical" ( $t=-1.438, p<.05)$, such that the non-canonical context conditions were processed faster than the "null" context conditions; (d) no significant interaction between "canonical" and RC Type $(t=1.245, p>.1)$ such that there was an ORC advantage in the canonical context condition; but a significant interaction between "non-canonical" and $\mathrm{RC}$ Type $(t=2.928, p<.01)$, such that there was an SRC advantage in the non-canonical context.

Analysis on the mainverb +1 region revealed (a) no significant main effect of RC Type $(t=-$ $0.842, p>.5)$, such that SRCs were processed slower than ORCs but not significant in the "null" context; (b) a significant main effect of "canonical" $(t=-4.572, p<.001)$, such that the canonical context conditions were processed faster than the "null" context conditions; (c) a significant main effect of "non-canonical" $(t=-4.465, p<.001)$, such that the non-canonical context conditions were processed faster than the "null" context conditions; (d) but no significant interaction between "canonical" and RC Type $(t=0.795, p>.1)$ and no interaction between "noncanonical" and RC Type $(t=0.351, p>.5)$.

3. Discussion. Table 1 summarizes the predictions of the different priming accounts for different context conditions and the actual observed results.

\begin{tabular}{|c|c|c|c|}
\hline Contexts & \multicolumn{2}{|c|}{ Priming accounts and their predictions } & Actual Observed Results \\
\hline \multirow{3}{*}{ canonical } & Thematic & ORC advantage & mostly an ORC advantage \\
\hline & & a larger SRC advantage & (except a SRC advantage on the \\
\hline & Syntactic & ORC advantage & $V N / N V$ region) \\
\hline \multirow{3}{*}{ non-canonical } & & & mostly an ORC advantage \\
\hline & $\mathrm{VP}$ & definitely not an ORC advantage & (except a SRC advantage on the \\
\hline & Syntactic & definitely not an ORC advantage & mainverb region) \\
\hline \multirow{3}{*}{ "null" } & Thematic & SRC advantage & ORC advantage \\
\hline & & SRC advantage & \\
\hline & Syntactic & SRC advantage & \\
\hline
\end{tabular}

Table 1: Predictions of the three different priming accounts for the three different context conditions and the actual observed results 
The results of this experiment replicated the ORC advantage in supportive canonical contexts, on the relativizer de region, the de+headnoun region, and the mainverb region, consistent with Vasishth et al. (2013) where a significant ORC advantage was found on the relativizer de and a marginally significant ORC advantage on the headnoun region. The results are also consistent with Lin $(2010,2014)$, who found a significant ORC advantage on the two regions after the headnoun. The ORC advantage on the relativizer de region could be interpreted as that the supportive contexts reduced the integration cost by priming the participants to expect and therefore process the RCs early.

However, the finding of a significant ORC advantage in "null" context conditions on the relativizer de region and the mainverb region is inconsistent with the majority of previous studies that have found a SRC advantage when the sentences containing RCs are presented without a context. This result is hard to reconcile with previous findings of a SRC advantage. The main difference between the "null" context condition in this study and the null context conditions in previous studies is that the "null" context condition in this study is not completely null, in that there is a context introducing the situation/event (most of the time also including the NPs), thus being a more natural reading than a completely null context condition, where there is no preceding context preceding the critical sentence with the RC.

The insignificance of the interactions between RC Type and "canonical" on all the regions is inconsistent with the findings in Lin $(2010,2014)$, who found no significant main effect of context or interaction between RC type and context on the first region after the headnoun. Yet he did find a significant interaction between RC type and context on the second region after the headnoun, such that the canonical context was shown to induce a significant ORC advantage as compared to a non-canonical context where there was no significant difference between SRCs and ORCs. Because Lin $(2010,2014)$ explained the finding of an ORC advantage on the second region after the headnoun only in the canonical context conditions in terms of thematic pattern priming, the same account cannot explain the significance of an ORC advantage in the "null" context conditions in this study.

There were discrepancies between predictions and the actual results for each priming account. For Chinese, all three accounts would incorrectly predicted a SRC advantage in the "null" context conditions, where an ORC advantage was actually found on the $d e, d e$ theadnoun, mainverb, and mainverb +1 regions, and also incorrectly predicted that there would not be an ORC advantage in the non-canonical context conditions, where an ORC advantage was indeed statistically reliable in the $d e$ and $d e+$ headnoun regions. The thematic pattern priming account and the syntactic position sequence priming account correctly predicted an ORC advantage in the canonical context conditions, whereas the VP constituent priming account incorrectly predicted a larger SRC advantage instead.

The significance of an ORC advantage in the "null" context in Chinese, and the insignificance of the interaction between RC Type and "canonical" in Chinese, together suggested that it was not the thematic pattern, or the syntactic position sequence, or the verb phrase constituent alone that was priming an ORC advantage in Chinese RC processing with a context. If we agree, based on the majority of the previous findings, that there is an SRC advantage when the RCs are processed without a context (null context), the finding of an ORC advantage in "null" contexts in this current study demonstrated that some other kind of factor was influencing the RC complexity effect and that with a preceding context, the frequency effects of SRCs and ORCs is reduced, or even reversed, as compared to in a null context condition. 
One possible explanation is that inclusion of a context makes the critical sentences with a $\mathrm{RC}$ more natural to process. Because all conditions in this experiment identify a set of referents that can be picked out by the RC, the RC complexity effect becomes smaller. An alternative explanation is the priming of the thematic role or grammatical function of the NPs in the "null" contexts preceding the encountering of the relative clause in the critical target sentence. Reanalysis of the items revealed that out of the 16 items in the "null" context conditions, 15 introduced the NP inside the relative clause in the critical target sentence, 14 of which were introduced uniquely as an AGENT/subject in the "null" context, thus favoring the ORC processing. For example, 'the TA' was the AGENT/subject in the sentence 'the TA gave the students some exercises for them to practice' in (7). The NP inside an ORC "the student that the TA opposes" is the AGENT/subject, which is consistent with its thematic role or grammatical function in the preceding context sentence. The NP inside a SRC "the student that opposes the TA" is the PATIENT/object, which is inconsistent with its thematic role or grammatical function in the preceding context. This explanation is tentative, since we did not intentionally control for the thematic roles of the NPs in the "null" contexts. Another possibility is topic prominence; note that the ORCs start with the NPs, which were introduced as the AGENT/subject in the "null" contexts, whereas the SRCs start with a verb. A direction for future research would be strictly counterbalance the thematic role and grammatical function of the NPs to test if this is indeed the case. The contexts used in this study and in the previous studies on RC processing in context were successful with respect to making $\mathrm{RC}$ processing more natural. However, since there was a lot of information, not only the events, but also the different NPs in the context sentences, the cognitive load might have been harder for participants to process, thus reducing the precision of measuring the processing of the target RC sentences. The finding that none of the three priming accounts could sufficiently explain all the results in Chinese does not preclude the possibility of the interplay of different kinds of priming, thus making the results harder to explain by means of one of them alone.

4. Conclusion. This experiment on Chinese RC processing in context has been able to show: (a) that contexts preceding RCs can influence the RC subject/object asymmetry effect; (b) that contexts preceding RCs do not influence the RC asymmetry effect in terms of any of the three different priming accounts that have been proposed by Lin $(2010,2014)$ or Fedorenko et al. (2012); (c) that any context that provides appropriate discourse for a RC can facilitate RC processing; and (d) that something else from the preceding contexts, possibly thematic roles of the specific NPs inside the RCs, is really playing a role in participants' subsequent processing of the RCs.

\section{References}

Bates, Douglas, Martin Maechler, Ben Bolker \& Steven Walker. 2014. Ime4: Linear mixedeffects models using Eigen and S4. R package version 1.1-7, http://CRAN.Rproject.org/package $=1$ me4.

Fedorenko, Evelina, Steve Piantadosi \& Edward Gibson. 2012. Processing Relative Clauses in Supportive Contexts. Cognitive Science 36(3). 471-497. http://dx.doi.org/10.1111/j.15516709.2011.01217.x.

Gennari, Silvia P. \& Maryellen C. MacDonald 2008. Semantic indeterminacy in object relative clauses. Journal of Memory and Language 58(2). 161-187.

http://dx.doi.org/10.1016/j.jml.2007.07.004. 
Gibson, Edward. 1998. Linguistic complexity: locality of syntactic dependencies. Cognition 68(1). 1-76. http://dx.doi.org/10.1016/S0010-0277(98)00034-1.

Gibson, Edward. 2000. The dependency locality theory: A distance-based theory of linguistic complexity. In Y. Miyashita, A. Marantz \& W. O’Neil (Eds.), Image, language, brain. 95126. Cambridge, MA: MIT Press.

Gibson, Edward, Timothy Desmet, Daniel Grodner, Duane Watson \& Kara Ko. 2005. Reading relative clauses in English. Cognitive Linguistics 16(2). 313-353. http://dx.doi.org/10.1515/cogl.2005.16.2.313.

Gibson, Edward \& H. H. Iris Wu. 2013. Processing Chinese relative clauses in context. Language and Cognitive Processes 28(1-2). 125-155. http://dx.doi.org/10.1080/01690965.2010.536656.

Hsiao, Franny \& Edward Gibson. 2003. Processing relative clauses in Chinese. Cognition 90(1). 3-27. http://dx.doi.org/10.1016/S0010-0277(03)00124-0.

Hsiao, Yaling \& Maryellen C. Macdonald. 2013. Experience and generalization in a connectionist model of Mandarin Chinese relative clause processing. Frontiers in Psychology 4. 767. http://dx.doi.org/10.3389/fpsyg.2013.00767.

Jäger, Lena, Zhong Chen, Qiang Li, Chien-Jer Charles Lin \& Shravan Vasishth. 2015. The subject-relative advantage in Chinese: Evidence for expectation-based processing. Journal of Memory and Language 79-80. 97-120. http://dx.doi.org/10.1016/j.jml.2014.10.005.

Just, Marcel A., Patricia A. Carpenter \& Jacqueline D Woolley. (1982). Paradigms and processes in reading comprehension. Journal of Experimental Psychology: General 111(2). 228-238. http://dx.doi.org/10.1037/0096-3445.111.2.228.

Keenan, Edward L. \& Bernard Comrie. 1977. Noun Phrase Accessibility and Universal Grammar. Linguistic Inquiry 8(1). 63-99.

Levy, Roger. 2008. Expectation-based syntactic comprehension. Cognition 106(3). 1126-1177. http://dx.doi.org/10.1016/j.cognition.2007.05.006.

Lewis, Richard L. \& Shravan Vasishth. 2005. An Activation - Based Model of Sentence Processing as Skilled Memory Retrieval. Cognitive Science 29(3). 375-419. http://dx.doi.org/10.1207/s15516709cog0000 25.

Lin, Chien-Jer Charles. 2010. Comprehending Chinese relative clauses in context: Thematic patterns and grammatical functions. In Lauren Eby Clemens \& Chi-Ming Louis Liu (eds.), Proceedings of the 22rd North American Conference on Chinese Linguistics (NACCL-22) and the 18th International Conference on Chinese Linguistics (IACL-18). Vol. 1. 413-428. Cambridge, MA: Harvard University.

Lin, Chien-Jer Charles. 2014. Effect of thematic order on the comprehension of Chinese relative clauses. Lingua 140. 180-206. http://dx.doi.org/10.1016/j.lingua.2013.12.003.

Lin, Chien-Jer Charles \& Thomas G. Bever. 2006. Subject preference in the processing of relative clauses in Chinese. In Donald Baumer D, David Montero \& Michael Scanlon (eds.), Proceedings of the 25th West Coast Conference on Formal Linguistics. 254-260. Sommerville, MA: Cascadilla Proceedings Project.

MacDonald, Maryellen C., Neal J. Pearlmutter \& Mark S. Seidenberg. 1994. Lexical Nature of Syntactic Ambiguity Resolution. Psychological Review 101(4). 676-703. http://dx.doi.org/10.1037/0033-295X.101.4.676.

MacDonald, Maryellen C. \& Morten H. Christiansen. 2002. Reassessing Working Memory: Comment on Just and Carpenter (1992) and Waters and Caplan (1996). Psychological Review 109(1). 35-54; discussion 55. http://dx.doi.org/10.1037/0033-295X.109.1.35. 
MacWhinney, Brian. 1977. Starting Points. Language 53(1). 152-168. http://dx.doi.org/10.2307/413059.

MacWhinney, Brian. 1982. Basic syntactic processes. In Stan Kuczaj (ed.), Syntax and semantics (1). Language acquisition. 73-136. Hillsdale, NJ: Erlbaum.

MacWhinney, Brian \& Csaba Pléh. 1988. The processing of restrictive relative clauses in Hungarian. Cognition 29(2). 95-141. http://dx.doi.org/10.1016/0010-0277(88)90034-0.

O’Grady, William D. 1997. Syntactic development. Chicago, IL: Chicago University Press.

Packard, Jerome L., Zhang Ye \& Xiaolin Zhou. 2011. Filler-gap processing in Mandarin relative clauses: Evidence from event-related potentials. In Hiroko Yamashita, Yuki Hirose \& Jerome Lee Packard (eds.), Processing and producing head-final structures. 219-240. Dordrecht, New York: Springer.

R Core Team. 2014. R: A language and environment for statistical computing. R Foundation for Statistical Computing, Vienna, Austria. http://www.R-project.org/.

Reali, Florencia \& Morten H. Christiansen. 2007. Processing of relative clauses is made easier by frequency of occurrence. Journal of Memory and Language 57(1). 1-23. http://dx.doi.org/10.1016/j.jml.2006.08.014.

Tabor, Whitney, Cornell Juliano \& Michael K. Tanenhaus. 1997. Parsing in a Dynamical System: An Attractor-based Account of the Interaction of Lexical and Structural Constraints in Sentence Processing. Language and Cognitive Processes 12(2). 211-271. http://dx.doi.org/10.1080/016909697386853.

Traxler, Matthew J., Robin K. Morris \& Rachel E. Seely. 2002. Processing Subject and Object Relative Clauses: Evidence from Eye Movements. Journal of Memory and Language 47(1). 69-90. http://dx.doi.org/10.1006/jmla.2001.2836.

Traxler, Matthew J., Rihana S. Williams, Shelley A. Blozis \& Robin K. Morris. 2005. Working memory, animacy, and verb class in the processing of relative clauses. Journal of Memory and Language 53(2). 204-224. http://dx.doi.org/10.1016/j.jml.2005.02.010.

Vasishth, Shravan, Zhong Chen, Qiang Li \& Gueilan Guo. 2013. Processing Chinese relative clauses: evidence for the subject-relative advantage. PLOS ONE 8(10). 1-15. http://dx.doi.org/10.1371/journal.pone.0077006.

Warren, Tessa \& Edward Gibson. 2002. The influence of referential processing on sentence complexity. Cognition 85(1). 79-112. http://dx.doi.org/10.1016/S0010-0277(02)00087-2.

Wu, Fuyun, Elsi Kaiser \& Elaine Andersen. 2012. Animacy effects in Chinese relative clause processing. Language and Cognitive Processes 27(10). 1489-1524. http://dx.doi.org/10.1080/01690965.2011.614423.

$\mathrm{Xu}, \mathrm{Yi}$. 2014. Processing relative clauses in Chinese as a second language. Second Language Research 30(4). 439-461. http://dx.doi.org/10.1177/0267658313511485.

Yun, Jiwon, Zhong Chen, Tim Hunter, John Whitman \& John Hale. 2015. Uncertainty in processing relative clauses across East Asian languages. Journal of East Asian Linguistics 24(2). 113-148. http://dx.doi.org/10.1007/s10831-014-9126-6. 